\section{Cardiotrophin workout}

\section{By Lev Osherovich, Senior Writer}

A team at the University of Navarra has shown that obesity and metabolic syndrome can be treated with cardiotrophin-1, a cytokine that regulates energy utilization and appetite. ${ }^{1}$ The findings open up a new indication for university spinout Digna Biotech S.L., which is developing the cytokine as a cytoprotective agent in liver transplantation.

Cardiotrophin-1 (CT-1) is a member of the IL-6 family of cytokines. According to Jesús Prieto, the compound "drew our attention about eight years ago because of its cytoprotective activities in hepatic indications."

Prieto is a professor of medicine and director of hepatology and gene therapy at the university's Centre for Applied Medical Research (CIMA).

Previous work had shown that other IL-6 family members could influence metabolism. ${ }^{2}$ Those findings prompted Prieto's team to examine the metabolic effects of deleting Ct-1 in mice.

According to Prieto, the current study shows "that CT-1 has an effect on metabolic activity. CT-1 is a main regulator of energy homeostasis, increasing whole-body oxygen consumption."

Indeed, Ct-1 knockout mice spontaneously developed the signs of metabolic syndrome, including obesity, dyslipidemia and hypertrophied white adipose tissue, whereas wild-type controls did not.

The knockout mice ate normal amounts of food but gained weight because they were unable to burn off excess energy.

"We found that Ct-1-deficient mice exhibit a significant reduction in energy expenditure and in turn develop obesity and hypercholesterolemia," said Prieto.

Conversely, wild-type mice treated with recombinant Ct-1 underwent a rapid uptick in metabolism and showed greater lipid oxidation and glucose utilization than saline-treated animals.

In a mouse model of obesity and metabolic syndrome, chronic treatment with recombinant $\mathrm{Ct}-1$ reduced blood glucose levels, shrank white adipose tissue and led to lower food intake and decreased body weight compared with saline treatment.

Data were reported in Cell Metabolism.

The precise mechanism by which CT-1 exerts its effects on body weight is unknown, but Prieto thinks the cytokine alters a range of physiological processes involving glucose and lipid metabolism, appetite and behavior.

"We have not yet identified the main target of CT-1," he said. He noted that the cellular receptor for CT-1-leukemia inhibitor factor receptor- $\alpha$ (LIFR; CD118) - is expressed in a range of tissues, including heart, liver and white adipose. Prieto said it remains unclear which of these tissues is responsible for the weight loss caused by recombinant CT-1.

In addition to narrowing down the tissues responsible for CT-1's metabolic effects, the team plans to optimize the dosage of CT-1 in preclinical models of obesity and metabolic syndrome.

\section{Energizer}

Digna hopes to move Prieto's work into preclinical development for metabolic indications once it finds a clinical development partner.

"Energy expenditure is an underexplored area" in metabolic disease therapy, said Lou Tartaglia, CSO of Rhythm Pharmaceuticals Inc. But historically, compounds that stimulate energy utilization have had overly broad effects on cellular metabolism and have run into safety issues, he added.

"It's been tried before with 2,4-dinitrophenol, which was a disaster, and with high doses of growth hormone, but these drugs didn't have the kind of safety that's required," he said.

2,4-Dinitrophenol, which disrupts the proton gradient that powers mitochondria, underwent clinical testing by Stanford University researchers as a weight loss agent in the 1930s but proved toxic at high doses. The compound continues to be used illicitly for rapid weight loss.

Rhythm's RM493, a peptide agonist of melanocortin 4 receptor (MC4R), is in preclinical development for obesity, and the company hopes to have the compound in the clinicl later this year. The compound modulates appetite and energy expenditure.

\section{Chronic issues}

Digna already has CT-1 in Phase I trials to prevent acute hepatotoxicity in liver transplant, in which the cytokine is administered during a brief window of time. For metabolic indications, it likely would be necessary to deliver CT-1 chronically, and whether the compound is well tolerated under such a regime will require further preclinical testing.

Prior work has hinted at negative cardiovascular safety signals associated with CT-1. University of Otago researchers have previously shown that CT-1 levels are increased in patients with hypertension. ${ }^{3}$ In addition, Roche's Genentech Inc. unit demonstrated that CT-1 injections led to cardiac hypertrophy in mice. ${ }^{4}$

Tartaglia thinks CT-1-induced myocardial remodeling could either be pathological or a benign response to increased energy utilization, depending on the dosing regimen. But demonstrating that such effects do not constitute a safety risk will be challenging, he said. 


\section{ANALYSIS}

"An energy expenditure drug could lead to improved cardiovascular function," said Tartaglia. "The issue with regulatory agencies is that you really have to prove that changes in the heart are for the good, not the bad."

Digna CEO Pablo Ortiz and Prieto noted that mice receiving daily doses of Ct-1 for two weeks did not show any cardiovascular anomalies, but they acknowledged that longer term cardiovascular toxicology studies are needed.

In 2009, Digna and partner Biotecnol S.A. in-licensed Genentech's IP covering the use of CT-1 in liver indications.

Digna has licensed new patents filed by the CIMA team on the use of CT-1 in metabolic disease.

The company has raised about $€ 34$ million ( $\$ 48.7$ million) in government funding, private equity and revenue from contract research since 2003. The company's most advanced product is P144, a topical formulation of transforming growth factor- $\beta 1$ inhibitor peptide 114, which is in Phase II testing for scleroderma and is partnered with Isdin S.A.
Osherovich, L. SciBX 4(33); doi:10.1038/scibx.2011.922

Published online Aug. 25, 2011

\section{REFERENCES}

1. Moreno-Allaga, M.J. et al. Cell Metab.; published online Aug. 3, 2011; doi:10.1016/j.cmet.2011.05.013

Contact: Matilde Bustos, University of Navarra, Pamplona, Spain e-mail: mbustos@unav.es

Contact: Jesús Prieto, same affiliation as above e-mail: mbustos@unav.es

2. Tilg, H. \& Moschen, A.R. Nat. Rev. Immunol. 6, 772-783 (2006)

3. Bordet, T. et al. J. Clin. Invest. 104, 1077-1085 (1999)

4. Jin, H. et al. Cytokine 8, 920-926 (1996)

COMPANIES AND INSTITUTIONS MENTIONED

Biotecnol S.A., Oeiras, Portugal

Digna Biotech S.L., Madrid, Spain

Genentech Inc., South San Francisco, Calif.

Isdin S.A., Barcelona, Spain

Roche (SIX:ROG; OTCQX:RHHBY), Basel, Switzerland

Rhythm Pharmaceuticals Inc., Boston, Mass.

Stanford University, Stanford, Calif.

University of Navarra, Pamplona, Spain

University of Otago, Otago, New Zealand 